\title{
PREDICTION OF CONFINEMENT EFFECTS ON THE NUMBER OF COVID-19 OUTBREAK IN ALGERIA
}

\author{
Ali Moussaoui ${ }^{1, *}$ And Pierre Auger ${ }^{2,3}$
}

\begin{abstract}
The first case of coronavirus disease 2019 (COVID-19) in Algeria was reported on 25 February 2020. Since then, it has progressed rapidly and the number of cases grow exponentially each day. In this article, we utilize SEIR modelling to forecast COVID-19 outbreak in Algeria under two scenarios by using the real-time data from March 01 to April 10, 2020. In the first scenario: no control measures are put into place, we estimate that the basic reproduction number for the epidemic in Algeria is 2.1, the number of new cases in Algeria will peak from around late May to early June and up to $82 \%$ of the Algerian population will likely contract the coronavirus. In the second scenario, at a certain date $T$, drastic control measures are taken, people are being advised to self-isolate or to quarantine and will be able to leave their homes only if necessary. We use $S E I R$ model with fast change between fully protected and risky states. We prove that the final size of the epidemic depends strongly on the cumulative number of cases at the date when we implement intervention and on the fraction of the population in confinement. Our analysis shows that the longer we wait, the worse the situation will be and this very quickly produces.
\end{abstract}

Mathematics Subject Classification. 92B05, 92C60.

Received April 13, 2020. Accepted June 29, 2020.

\section{INTRODUCTION}

The cumulative number since 25 February, when the first case was diagnosed, to present day, is 1761 cases, including 529 from the city of Blida and 310 from Algiers. Since then, the infection has become more virulent, and the number of cases has been increasing. It should be noted that screening remains very low in relation to the means available. Figure 1 shows the cumulative number of detected cases of coronavirus in Algeria between March 01 and April 10, 2020. Between these two dates, the cumulative number of cases increased from 03 to 1761. The spread of the epidemic adjusts very well (which is well known in epidemiology) with an exponential growth for the number of contaminated cases $I$ over time:

$$
I(t)=I_{0} e^{\lambda t}
$$

Keywords and phrases: COVID-19, SEIR compartmental model, basic reproduction number, time scales, aggregation of variables.

${ }^{1}$ Department of Mathematics, Faculty of Sciences, University of Tlemcen, Algeria.

2 UMMISCO, Centre IRD d'Ile de France, Bondy, France.

3 Sorbonne Universités, Paris, France.

* Corresponding author: ali.moussaoui@univ-tlemcen.dz 


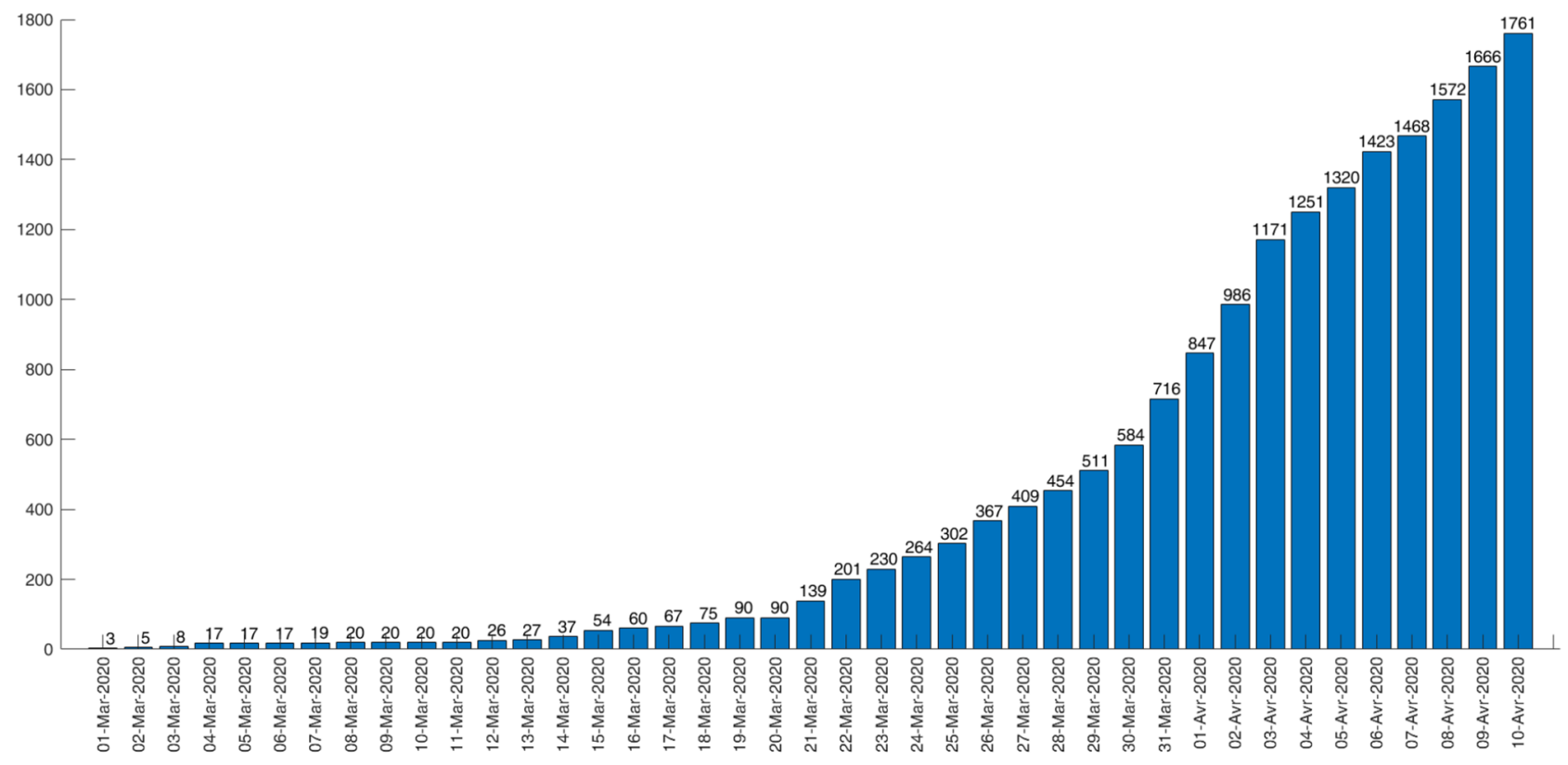

Figure 1. COVID-19 cases in Algeria, [7, 14].

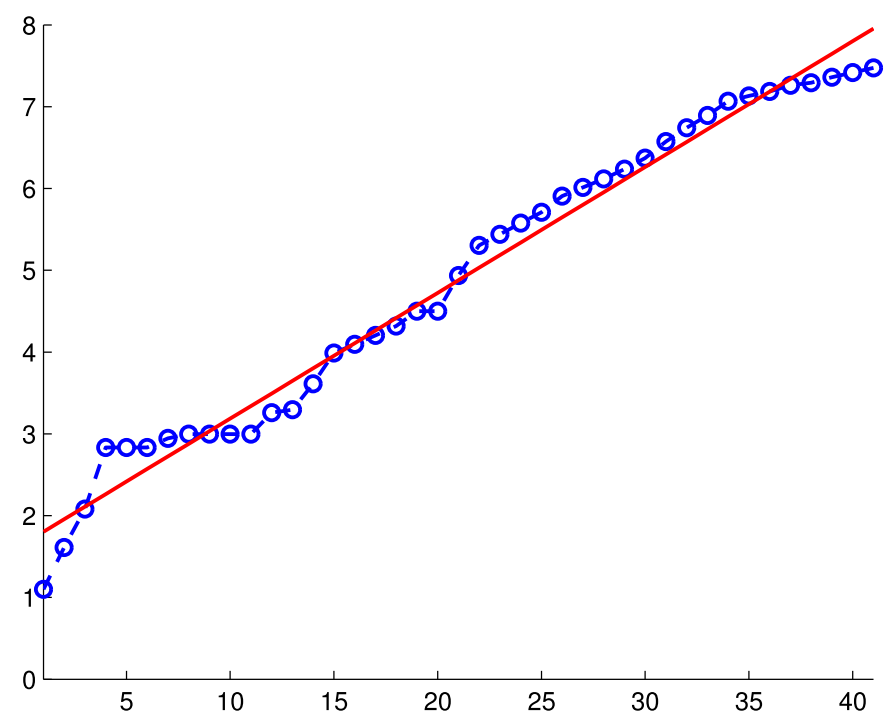

Figure 2. Neperian logarithm of the cumulative number of cases and linear regression lines.

By using a least-squares fit for the exponential function (1.1) for the number of contaminated cases with the data shown in Figure 2, we find for Algeria $\lambda \approx 0.16$. If this rhythm of growth continues, Algeria will be faced with tens, even hundreds of thousands of cases in the space of a few weeks, including thousands of patients requiring intensive care. 


\section{THE MODEL WITHOUT INTERVENTION}

Multiple epidemiological models have been proposed to predict the spread of COVID-19 epidemic, we cite here the works of Liu et al. [10], where the authors developed a model of the COVID-19 epidemic in Wuhan, China. Magal and Webb [12] studied the spread of the epidemic in South Korea, Italy, France and Germany. Rong et al. [15] evaluated effects of delay in diagnosis on transmission of COVID-19. Kuniya [8] studied the prediction of the epidemic Peak of COVID-19 in Japan. Bacaër [3] studied a mathematical model of the beginning of the epidemic in France.

\subsection{Estimation of the $S E I R$ model parameters}

To model the dynamic of the disease and predict the number of COVID-19 cases in Algeria, we use a standard $S E I R$ model. We let $S(t)$, denote the fraction of individuals who are susceptible to the disease, that is, who are not (yet) infected at time $t, E(t)$ denotes the fraction of exposed or latent individuals that is, who are infected but not yet infectious at time $t, I(t)$ denotes the fraction of infected individuals, assumed infectious and able to spread the disease by contact with susceptible at time $t . R(t)$ corresponds to the fraction of cumulative number of known cases (infectious but confined at home, in hospital, recover or die from the disease) at time $t$.

The $S E I R$ model can be written as follows:

$$
\begin{aligned}
& \frac{\mathrm{d} S}{\mathrm{~d} t}=-\beta S(t) I(t), \\
& \frac{\mathrm{d} E}{\mathrm{~d} t}=\beta S(t) I(t)-k E(t), \\
& \frac{\mathrm{d} I}{\mathrm{~d} t}=k E-\alpha I, \\
& \frac{\mathrm{d} R}{\mathrm{~d} t}=\alpha I, \\
& S(t)+E(t)+I(t)+R(t)=1,
\end{aligned}
$$

where $\beta$ is the transmission rate per infectious individual, $k$ is the infection rate calculated by the inverse of the average incubation period, based on the previous studies $[8,9,16]$, we fix $1 / k=5$, and thus, $k=0.2 .1 / \alpha$ is the average time in compartment " $I$ " before isolation. It is more difficult to estimate $\alpha$ because, it depends not only on the biological characteristics of the virus but also on the promptness of isolation after symptom onset. Let's assume as in [3] that it's on the order of 1 day because when the patient senses the symptoms, he calls the medical center to come and take care of him on the same day.

Infected case data can then be used to estimate unknown epidemiological parameters, including the transmission rate $\beta$ and the basic reproduction ratio $\mathcal{R}_{0}$. In the case of the $S E I R$ model, the predicted exponential growth rate, can be derived from a solution of the linearized dynamics near the Disease-Free Equilibrium $(D F E)$ $(1,0,0,0)[11,18]$. The equations for $E$ and $I$ are decoupled and we obtain a linear system of the form

$$
\begin{aligned}
& \frac{\mathrm{d} E}{\mathrm{~d} t}=-k E+\beta I, \\
& \frac{\mathrm{d} I}{\mathrm{~d} t}=k E-\alpha I .
\end{aligned}
$$

The predicted exponential growth rate, $\lambda$ correspond to the largest eigenvalue of the Jacobian:

$$
J=\left[\begin{array}{cc}
-k & \beta \\
k & -\alpha
\end{array}\right]
$$


It can be shown that the growth of the number of infected cases is an exponential of the form $I(t) \sim I_{0} e^{\lambda t}$ where

$$
\lambda=\frac{-(k+\alpha)+\sqrt{(k-\alpha)^{2}+4 k \beta}}{2} .
$$

In addition, initially, $S \simeq 1$, thus, the incidence rate (number of new cases per day) $C=\beta S I$ also increases exponentially.

The basic reproduction number $\mathcal{R}_{0}$, which means the expected number of secondary cases produced by a single (typical) infection in a completely susceptible population [5] is calculated as the maximum eigenvalue of the next generation matrix $F V^{-1}[12]$, where

$$
J=\left[\begin{array}{cc}
0 & \beta \\
0 & 0
\end{array}\right], \quad V=\left[\begin{array}{cc}
k & 0 \\
-k & \alpha
\end{array}\right],
$$

then

$$
\mathcal{R}_{0}=\frac{\beta}{\alpha} .
$$

In order to determine the value of $\beta$ and $\mathcal{R}_{0}$ from the data, we use the classical method, see [3, 11]. From (2.9), express $\beta$ in terms of $\lambda$ and substitute it into $\mathcal{R}_{0}$, we get

$$
\beta=(\lambda+\alpha)\left(1+\frac{\lambda}{k}\right) \quad \text { and } \quad \mathcal{R}_{0}=\left(1+\frac{\lambda}{\alpha}\right)\left(1+\frac{\lambda}{k}\right)
$$

Thus, it is possible to estimate $\beta$ and $\mathcal{R}_{0}$ using the data set, we get,

$$
\mathcal{R}_{0} \simeq 2.1, \beta \simeq 2.1
$$

Following [3], adding (2.6) and (2.7), we obtain

$$
\frac{\mathrm{d}(E+I)}{\mathrm{d} t}=(\beta-\alpha) I=\left(\mathcal{R}_{0}-1\right) \frac{\mathrm{d} R}{\mathrm{~d} t} .
$$

Hence, at the beginning of the epidemic, we obtain the same result obtained in [3]:

$$
E(t)+I(t)+R(t) \approx \mathcal{R}_{0} R(t)
$$

\subsection{The final sizes of $S_{\infty}, R_{\infty}$ when no control measures are put into place}

The final size relation gives a relationship between the basic reproduction number and the size of the epidemic, that is the number of members of the population who are infected over the course of the epidemic [4].

Integration of the sum of the three equations (2.1), (2.2) and (2.3) from 0 to $\infty$ gives

$$
R_{\infty}=1-S_{\infty}=\alpha \int_{0}^{\infty} I(t) \mathrm{d} t
$$

Division of the equation (2.1) by $S$ followed by integration from 0 to $\infty$ gives

$$
-\log \frac{S_{\infty}}{S_{0}}=\beta \int_{0}^{\infty} I(t) \mathrm{d} t=\mathcal{R}_{0}\left(1-S_{\infty}\right) .
$$



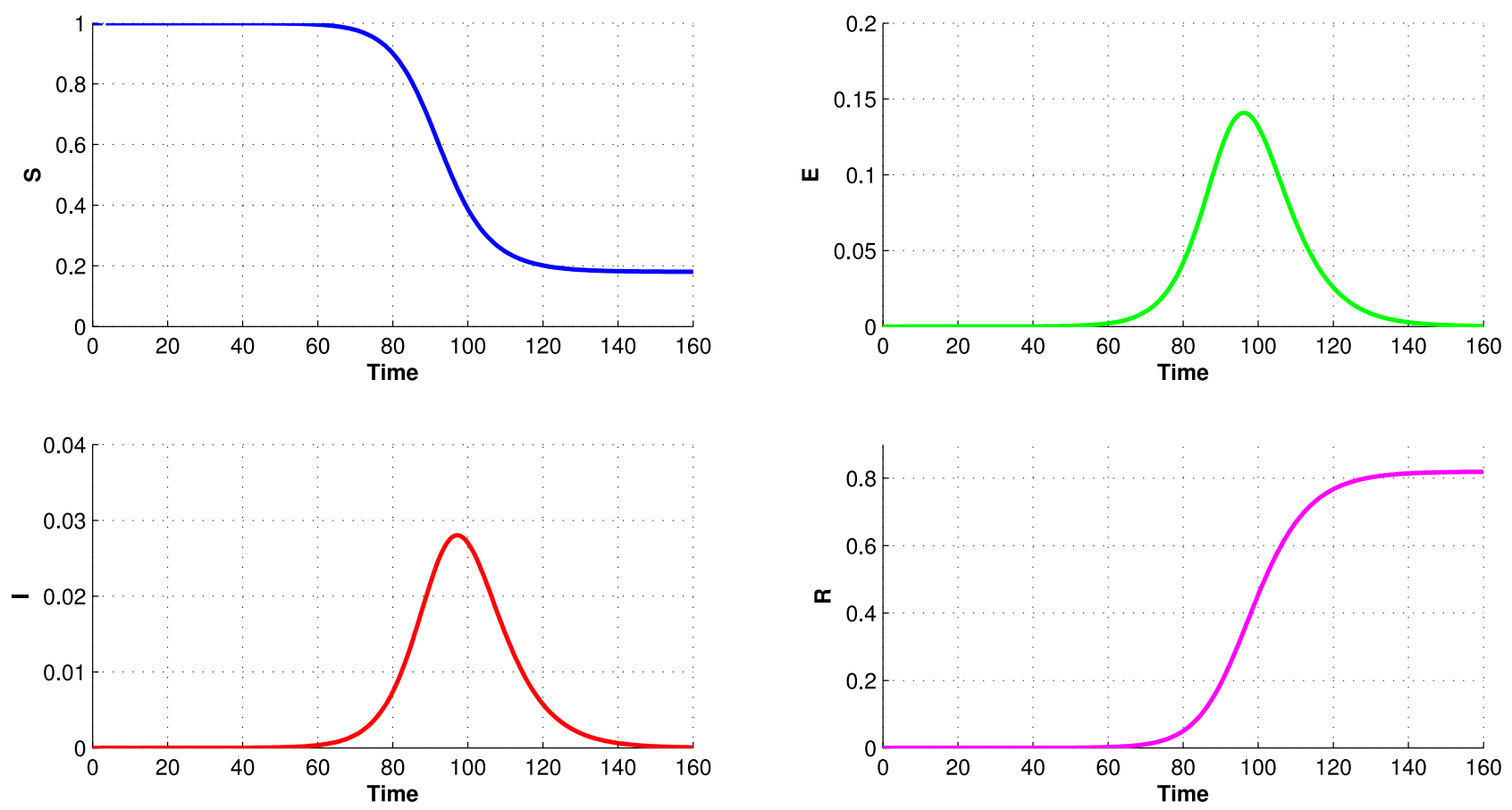

FiguRE 3. Solutions of SEIR system (2.1)-(2.5), $S_{0}=1-10^{-7}, E_{0}=0, I_{0}=10^{-7}, R_{0}=0$. The total resident population in Algeria reached 43 million.

Equation (2.15) gives implicitly $S_{\infty}$ and therefore also the final epidemic size $R_{\infty}=1-S_{\infty}$. At the beginning of the epidemic, the initial fraction of infected people $I_{0}$ is small compared with the population size, so $S_{0} \approx 1$, formula (2.15) can be rewritten using $S_{\infty}=1-R_{\infty}$ as

$$
1-R_{\infty} \approx e^{-\mathcal{R}_{0} R_{\infty}}
$$

The number of new cases in Algeria will peak from around late May to early June and up to $82 \%$ of the Algerian population will likely contract the coronavirus if no measures were imposed, see Figure 3.

An important aspect to take into account in the model concerns the proportion of asymptomatic infectious people. Taking $\alpha=1$ we assume that almost all infected are symptomatic and that they are isolated very quickly on average after one day. However, observations have shown that the proportion of asymptomatic patients could be around $50 \%$ and even more as it was reported on the cruise ship Diamond Princess, and more recently on the Charles de Gaulle aircraft carrier. Therefore, if $50 \%$ of the infected are asymptomatic with an average duration of infection of 7 to 10 days while the infected with symptoms are quickly isolated in 1 day, the average duration of stay in compartment $I$ will be a little more than 4 to 5 days with a $\alpha$ of about 0.2 .

Table 1 shows the variation in the basic reproduction rate of the epidemic as a function of the $\alpha$ parameter. For a residence time in the infectious compartment of 5 days, which corresponds to $\alpha=0.2$, the $R_{0}$ increases to take the value of 3.24 which corresponds to the estimated average value of $R_{0}$ in most cases.

Figure 4 shows that the choice of an $\alpha$ value of 0.1 , corresponding to a stay of 10 days in the infectious compartment, would lead to infection of almost the entire population in Algeria (blue curve) with an $R_{0}$ of 4.6 which seems overvalued. It seems more realistic to think that the value of the $\alpha$ parameter is around 0.2 , which corresponds to a length of stay of 5 days in the compartment $I$, and which leads to a more realistic situation with infection of $90 \%$ of the population in Algeria (green curve) and to a more realistic value of $R_{0}=3.24$. 
TABLE 1. Estimated parameters value for SEIR model (2.1)-(2.5).

\begin{tabular}{lllllllllll}
\hline$\alpha$ & 0.1 & 0.2 & 0.3 & 0.4 & 0.5 & 0.6 & 0.7 & 0.8 & 0.9 & 1 \\
$\beta$ & 0.46 & 0.64 & 0.82 & 1,008 & 1.188 & 1.36 & 1.54 & 1.72 & 1.90 & 2.1 \\
$R_{0}$ & 4.6 & 3.24 & 2.76 & 2.52 & 2.37 & 2.28 & 2.21 & 2.16 & 2.12 & 2.1 \\
\hline
\end{tabular}
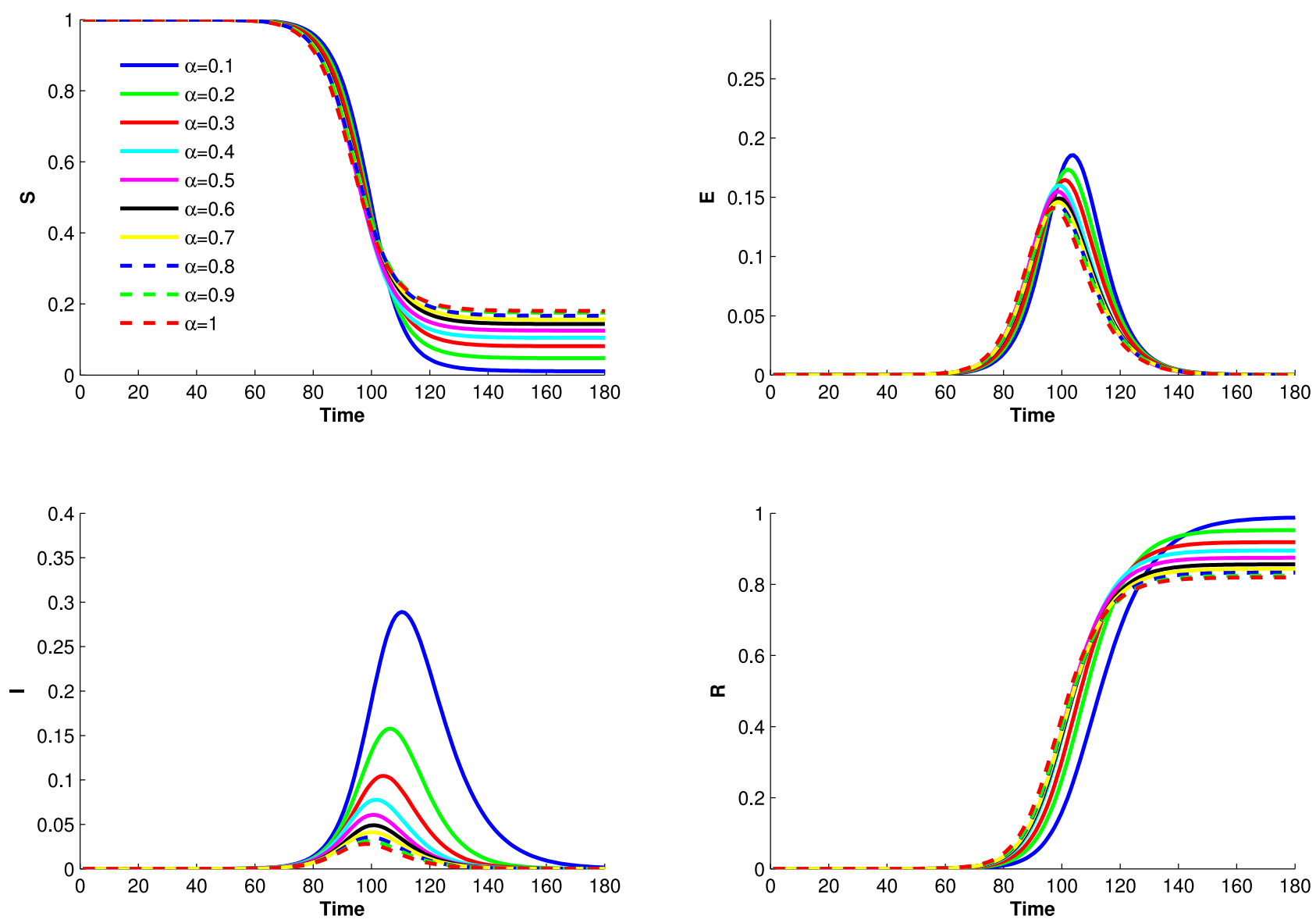

FiguRE 4. Impact of the average time in compartment $I$ before isolation on the solutions of $S E I R$ system (2.1)-(2.5).

\section{INTEGRATION OF INTERVENTION STRATEGIES}

In order to avoid this catastrophic situation and limit the exposure to the virus, partial "confinement" measures were imposed on some cities that have reported a highest number of contaminated cases. More people are being advised to self-isolate or to quarantine, they leave their homes only to buy essential items like food and medicine. To help in the evaluation, we opted for a simplified all or nothing model. We consider two individual states in which an average individual (representative of the entire population) who may be susceptible, exposed or infectious may find himself during the day. The first state corresponds to total protection in which the individual cannot be infected. The second state is a state where the individual is unprotected, that is to say with a transmission rate exactly identical to that of the epidemic before confinement. Thus, the states do not correspond to specific places. For example, state 1 is not associated only with the home where people are confined. Confined persons can go out of their house to play sport every morning in a park and never meet anyone and be in state 1 . They can go shopping in a shopping center every day wearing a fully effective mask 
and therefore be fully protected again in state 1 . As a result, a person in state 1 can be in various places. However, if the same person does not wear a mask in the shopping center, she is likely to be contaminated and is no more in state 1 but in state 2. Similarly, during the period of confinement, part of the population essential to operate vital services for the population (hospital services, police, security and cleaning of public places, minimum public transport etc) continues to move as before the epidemic. These people frequent many places every day where they can become infected. As soon as people are no longer completely protected, they are in state 2. Having no data on the places nor on the transmission rates, before and after confinement, we chose a simplified model with two extreme states, a fully protected state 1 and a state 2 with a maximum risk with a transmission rate identical to the one before the confinement. Then, the model is interested in the proportion of the time that an individual spends on average in state 1 and 2 during his day.

The model is a slow/fast model. We assume that all individuals travel daily to different locations. Even confined people go out almost each day to play sports, to buy basic necessities etc. The change of state takes place on the time scale of the day. This rapid model is coupled to a slow classic SEIR epidemic model. Indeed, the epidemic takes place over several weeks or even months, and the time scale of the epidemic model is therefore the month. The main output of the model is the fraction $v$ of its time that an average individual spends in fully protected state 1 and the complement $u=1-v$ in state 2 without any protection. Total confinement (lockdown) corresponds to all individuals in state $1, u=0$. The situation before the epidemic corresponds to all individuals in state $2, u=1$. An intermediate situation with partial confinement corresponds for example to an individual who would be in $20 \%$ of his time in state 2 of risk of major infection and $80 \%$ of his time in state 1 of total protection, $u=0.2$. It is important to clarify that the proportion of people in state 1 does not correspond exactly to the proportion of people confined, but rather corresponds to a gain in the total protection rate resulting from confinement. To be able to establish the link, it would be necessary to have reliable data on the frequentation rates of the various places with their transmission rates before and after confinement and to make a much more complex model. Without access to these data, we chose a minimal model where the individual changes state, from total protection to non-protection. It is clear that confinement modifies the frequency $v$ of time spent in state 1 of total protection. Very roughly, one can imagine that a very strict confinement of the population like that established in China where people absolutely couldn't get out of their homes and even in Algeria, increases $v$ more or less in the same proportion as the rate of confined persons. We generated different scenarios with varying values of these fractions in order to evaluate the effect of confinement on the spread of the coronavirus.

The susceptible individuals are divided into two sub-populations: Susceptible individuals in state 1 fully protected $\left(S_{1}\right)$, and $\left(S_{2}\right)$; Susceptible individuals in state 2 capable of contracting the disease and becoming themselves infectious. We use the same notations for exposed and infected individuals. Let $S(t)=S_{1}(t)+$ $S_{2}(t), E(t)=E_{1}(t)+E_{2}(t)$ and $I(t)=I_{1}(t)+I_{2}(t)$ be the fraction of total susceptible, exposed and infected individuals, respectively. All individuals, susceptible, exposed and infected $S_{1}, E_{1}$ and $I_{1}$ can change states daily, respectively, at rate $m_{2}$ by returning to a risk-free activity. The individuals can stop a safe activity at rate $m_{1}$ to carry out an activity where there is a risk of infection. Thus the time scale of the change of states of a typical individual is the day while the epidemic takes place on a time scale of several weeks even months. Therefore, we assume fast exchanges between the compartments with respect to the dynamics of the epidemics. Figure 5 shows how the disease is progressing from one sub-population to another. The diagram shows that individuals in state 1 cannot be infected. Contamination only occurs through contact between an infected individual and a susceptible one in a risky activity, state 2 . The exposed individuals do not even know that they have been infected and continue to change states as the susceptible ones. The same goes for asymptomatic infected people. Symptomatic infected people before seeing the doctor or going to the hospital continue to carry out various protected or risky activities. But as soon as the symptoms are declared, they are quickly isolated and go to compartment $R$.

The following complete model describes the time evolution of the epidemic:

$$
\frac{\mathrm{d} S_{1}}{\mathrm{~d} \tau}=m_{2} S_{2}-m_{1} S_{1}
$$




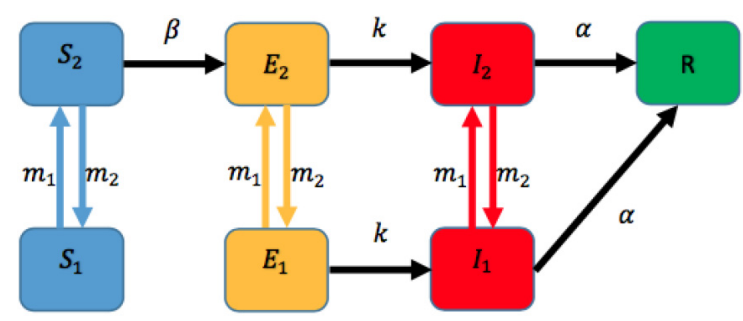

FiguRE 5. Flow diagram of the transmission dynamics of COVID-19.

$$
\begin{aligned}
\frac{\mathrm{d} S_{2}}{\mathrm{~d} \tau} & =m_{1} S_{1}-m_{2} S_{2}-\varepsilon\left(\beta S_{2}(t) I_{2}(t)\right) \\
\frac{\mathrm{d} E_{1}}{\mathrm{~d} \tau} & =m_{2} E_{2}-m_{1} E_{1}+\varepsilon\left(-k E_{1}\right) \\
\frac{\mathrm{d} E_{2}}{\mathrm{~d} \tau} & =m_{1} E_{1}-m_{2} E_{2}+\varepsilon\left(\beta S_{2}(t) I_{2}(t)-k E_{2}\right), \\
\frac{\mathrm{d} I_{1}}{\mathrm{~d} \tau} & =m_{2} I_{2}-m_{1} I_{1}+\varepsilon\left(k E_{1}-\alpha I_{1}\right) \\
\frac{\mathrm{d} I_{2}}{\mathrm{~d} t} & =m_{1} I_{1}-m_{2} I_{2}+\varepsilon\left(k E_{2}-\alpha I_{2}\right) \\
\frac{\mathrm{d} R}{\mathrm{~d} \tau} & =\varepsilon\left(\alpha I_{1}+\alpha I_{2}\right)
\end{aligned}
$$

where $\tau$ is the fast time, $t=\varepsilon \tau$ is the slow time and where $\varepsilon \ll 1$ is a small dimensionless parameter. The previous complete model can be reduced. As a first step, we consider the fast system which is obtained by setting $\varepsilon=0$ in the complete system. In our case, the fast equilibrium can be easily calculated and is given by:

$$
S_{1}^{*}=v S, \quad S_{2}^{*}=u S, \quad E_{1}^{*}=v E, \quad E_{2}^{*}=u E, \quad I_{1}^{*}=v I, \quad I_{2}^{*}=u I,
$$

where $v=\frac{m_{2}}{m_{1}+m_{2}}$ is the fraction of protected people due to confinement and $u=(1-v)$ is the fraction of individuals performing risky activities.

Using aggregation of variables methods [1,2], a reduced model is obtained by substituting the fast stable equilibrium into the complete system and by adding two equations for susceptible populations. This leads to the following aggregated model at the slow time which is valid when the reduced system is structurally stable and for small value of $\varepsilon \ll 1$ :

$$
\begin{aligned}
\frac{\mathrm{d} S}{\mathrm{~d} t} & =-\beta_{1} S(t) I(t), \\
\frac{\mathrm{d} E}{\mathrm{~d} t} & =\beta_{1} S(t) I(t)-k E(t), \\
\frac{\mathrm{d} I}{\mathrm{~d} t} & =k E-\alpha I, \\
\frac{\mathrm{d} R}{\mathrm{~d} t} & =\alpha I,
\end{aligned}
$$

where $\beta_{1}=u^{2} \beta$. This system is exactly the same as in the previous section except that the constant proportion $u$ appears in the equations via the parameter $\beta_{1}$.

The total number of infected individuals since the beginning of the epidemic can easily be calculated based on the date of confinement $T$. We can repeat the same study by integrating between $T$ and $\infty$, we obtain the 
following result:

$$
\log \frac{S(\infty)}{S(T)}=-u^{2} \mathcal{R}_{0}(R(\infty)-R(T))
$$

and this leads to the form

$$
1-R(\infty)=S(T) e^{-u^{2} \mathcal{R}_{0}(R(\infty)-R(T))}
$$

As control measures steps were taken at the beginning of the epidemic, we can combine (3.14) with (2.13) and we obtain

$$
1-R(\infty) \simeq\left(1-\mathcal{R}_{0} R(T)\right) e^{-u^{2} \mathcal{R}_{0}(R(\infty)-R(T))},
$$

If $u^{2} \mathcal{R}_{0}>1$, then the classical graphical argument [3,6] which consists in plotting the left and right members of equation (3.15) as a function of $R(\infty)$ shows that the solution $R(\infty)$ is not infinitesimal but close to the strictly positive solution of the equation

$$
1-R(\infty) \simeq e^{-u^{2} \mathcal{R}_{0} R(\infty)}
$$

If on the contrary $u^{2} \mathcal{R}_{0}<1$. As $0<R(\infty)-R(T)<1$, the approximation $e^{-x} \approx 1-x$ gives

$$
1-R(\infty) \simeq\left(1-\mathcal{R}_{0} R(T)\right)\left(1-u^{2} \mathcal{R}_{0}(R(\infty)-R(T)) .\right.
$$

Following [3], we obtain

$$
R(\infty) \simeq \mathcal{R}_{0} R(T) \frac{1-u^{2}}{1-u^{2} \mathcal{R}_{0}}
$$

When $u \rightarrow 0$, (corresponding to lockdown measures), the final size of the epidemic will be

$$
R(\infty) \simeq \mathcal{R}_{0} R(T)
$$

It is possible to calculate a threshold for the release of confinement making it possible to avoid a second epidemic peak. A simple way to find this threshold is to consider the effective reproduction rate of the epidemic $\mathcal{R}_{0}\left(T^{*}\right)$ at time $T^{*}$ of the release of confinement of the third phase and which is given by the following expression:

$$
R_{0}\left(T^{*}\right)=\left(u^{*}\right)^{2} \mathcal{R}_{0} S\left(T^{*}\right) .
$$

Here we note $u^{*}=u\left(T^{*}\right)$ which is the constant proportion of un-confined persons fixed at $t=T^{*}$. Those persons are free to move exactly as they did before the epidemic started. In order to avoid a secondary peak, we need an effective $\mathcal{R}_{0}\left(T^{*}\right)$ smaller than 1 which leads to the next condition:

$$
u^{*}=\frac{1}{\sqrt{\mathcal{R}_{0} S\left(T^{*}\right)}}
$$

It is good to note that the threshold depends on the proportion of susceptible at the time of deconfinement. If for example, in the case where $\alpha=0.2$ corresponding to an $R_{0}$ of 3.24 , there are $10 \%$ of people who have already been infected with covid-19, the threshold $u^{*}$ becomes equal to approximately $59 \%$. This level means 
TABLE 2. Estimated of $u^{*}$ with different values of $\alpha$.

\begin{tabular}{lllllllllll}
\hline$\alpha$ & 0.1 & 0.2 & 0.3 & 0.4 & 0.5 & 0.6 & 0.7 & 0.8 & 0.9 & 1 \\
$\beta$ & 0.46 & 0.64 & 0.82 & 1,008 & 1.188 & 1.36 & 1.54 & 1.72 & 1.90 & 2.1 \\
$R_{0}$ & 4.6 & 3.24 & 2.76 & 2.52 & 2.37 & 2.28 & 2.21 & 2.16 & 2.12 & 2.1 \\
$u^{*}$ & 0.49 & 0.59 & 0.64 & 0.66 & 0.68 & 0.7 & 0.71 & 0.72 & 0.725 & 0.73 \\
\hline
\end{tabular}

that we can allow $59 \%$ of the population to be completely free to carry out all risky activities as before the epidemic but while maintaining the remaining $41 \%$ of the population to be fully protected (see Tab. 2). In our model, this threshold means that a relatively large proportion of the population, around $60 \%$, can be authorized to be totally unconfined without any protective measures, while $40 \%$ must remain totally confined. However, a large-scale testing policy should also make it possible to rapidly isolate infected persons by removing them from the contamination chain, which is equivalent to taking a larger $\alpha$ value. If for example, in the case where $\alpha=0.5$ corresponding to an $R_{0}$ of 2.37 , there are $10 \%$ of people who have already been infected with covid-19, the threshold $u^{*}$ becomes equal to approximately $68 \%$. In our model, this threshold means that a relatively large proportion of the population, around $70 \%$, can be authorized to be totally unconfined without any protective measures, while $30 \%$ must remain totally confined. Furthermore, if protective measures with masks as well as social distancing are taken, they will also have an effect on the threshold of deconfinement by reducing importantly the rate of contact between individuals.

Knowing that a relatively large part of the population will continue to work in teleworking and that a part of the population in particular in establishments for the elderly will be confined and very protected, it appears possible to reach the threshold necessary to avoid a secondary peak after the lifting of the initial total containment. This aspect must be very seriously studied to limit the dramatic consequences of too strict confinement on economic activity.

\section{Discussion AND CONCLUSION}

We can predict what will happen in Algeria according to the date of our partial and total confinement. The blue curves in Figure 6a-d simulate what could happen without any intervention, the worst-case scenario, the number of new cases in Algeria will peak from around late May to early June and up to $82 \%$ of the Algerian population will likely contract the coronavirus. The other two curves (red and green) in Figure 6a correspond to the prediction if partial confinement will be carried out moderately end-April ( $v=10 \%$ in green) or severe $(v=50 \%$ in red).

Figure $6 \mathrm{~b}$ shows that the choice of the confinement date is very important. Indeed, an early date of partial confinement provokes a delay in the epidemic peak which is not desirable. From the economic point of view, we would favor a situation with an epidemic lasting less time in order to restart the economics faster. However, Figure $6 \mathrm{~b}$ also shows that an early date of confinement decreases the amplitude of the peak which is of course very desirable from the point of view of hospitals and medicists which would be confronted to less patients at the same time and would be able to take care of all of them. Therefore, there is a balance to find between economic and medical needs. This balance is to be considered by decision makers.

The curves in red in Figure 6c and d correspond to the lockdown. However, in the case of a lockdown at $T=80$, when people are going to be unconfined, a second peak with large amplitude can occur if the proportion of unconfined persons is too large, that is if $u>u^{*}$. Figure $6 \mathrm{c}$ shows two cases of unsuccessful unconfinement, ( $u=0.8$ at $T=90$ and at $T=100$ ), after lockdown with occurrence of a second peak. Therefore, it is needed to unconfine at a level below the threshold $u<u^{*}$ in order to control definitively the epidemic. Figure $6 \mathrm{~d}$ shows such a case of lockdown at $T=80$ followed by successful unconfinement at $T=100$ without resurgence of the epidemic. Our simulation shows that with confinement of only $40 \%$ of the population, it is possible to avoid a second epidemic peak. Taking into account, the proportion of people already contaminated by the first peak and cured, confining people at risk and elderly could be enough to prevent a resurgence of the epidemic after the first peak. 


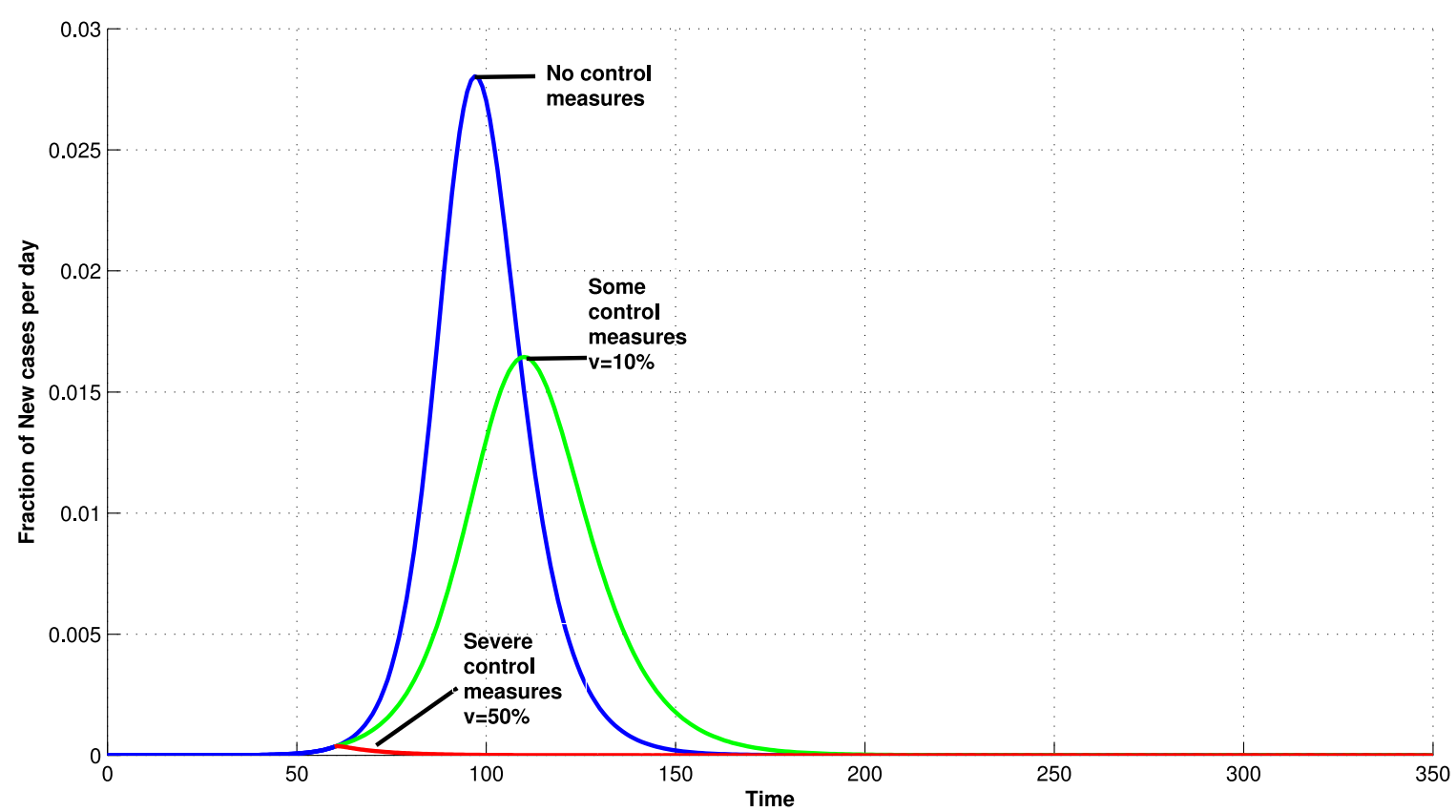

(a)

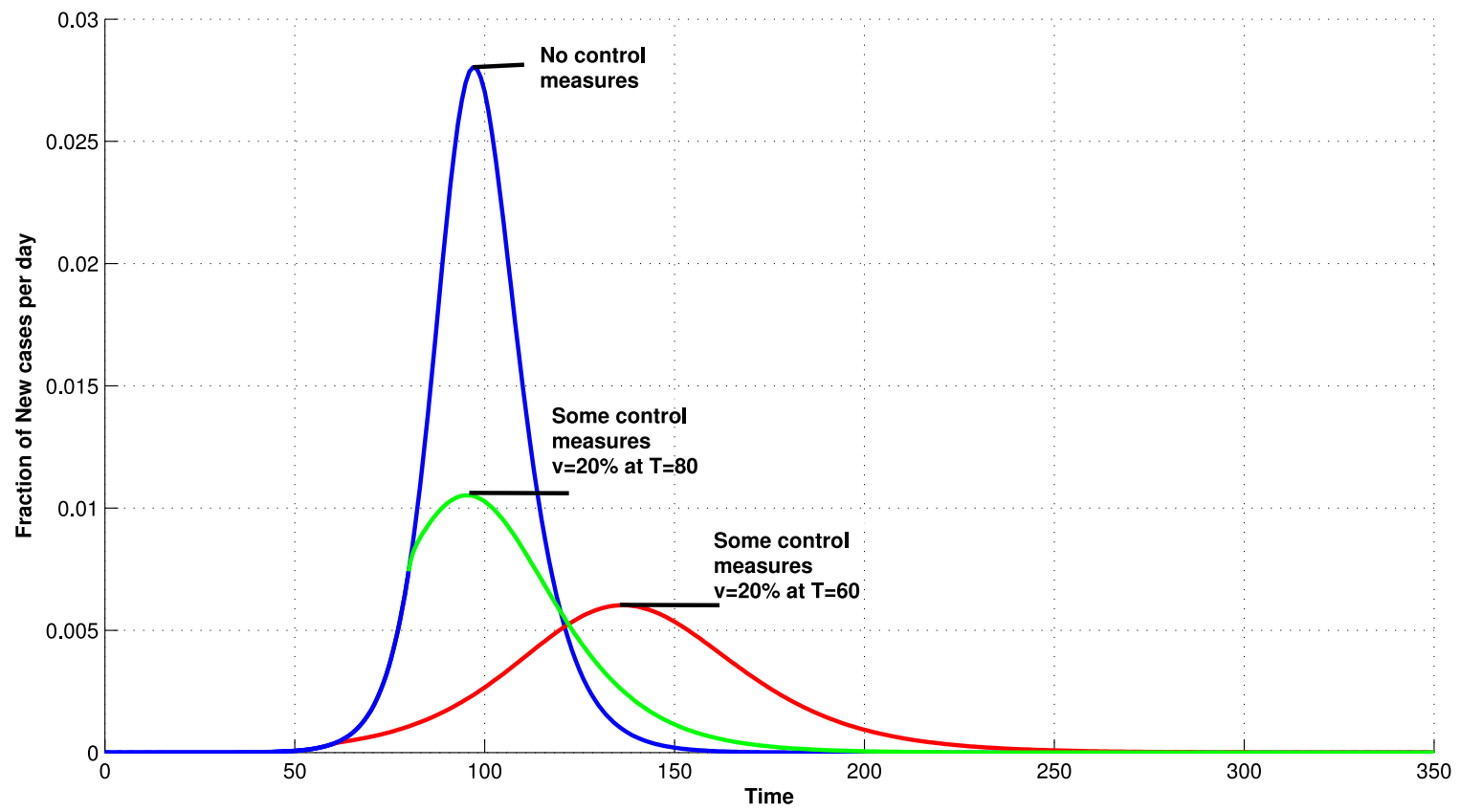

(b)

Figure 6. How control measures could slow the outbreak. Without action (blue). (a) Some control measures $(v=10 \%$, green), severe control measures $(v=50 \%$, red). (b) Some control measures at $T=60(v=20 \%$, red $)$, some control measures at $T=80(v=20 \%$, green). (c) Lockdown $(v=100 \%$, red $)$ at $T=80$ followed with unsuccessful unconfinement, $u=0.8$ either at $T=90$ or at $T=100$. (d) Lockdown $(v=100 \%$, red) at $T=80$ followed with successful unconfinement at $T=90(u=0.6$, green $)$. 


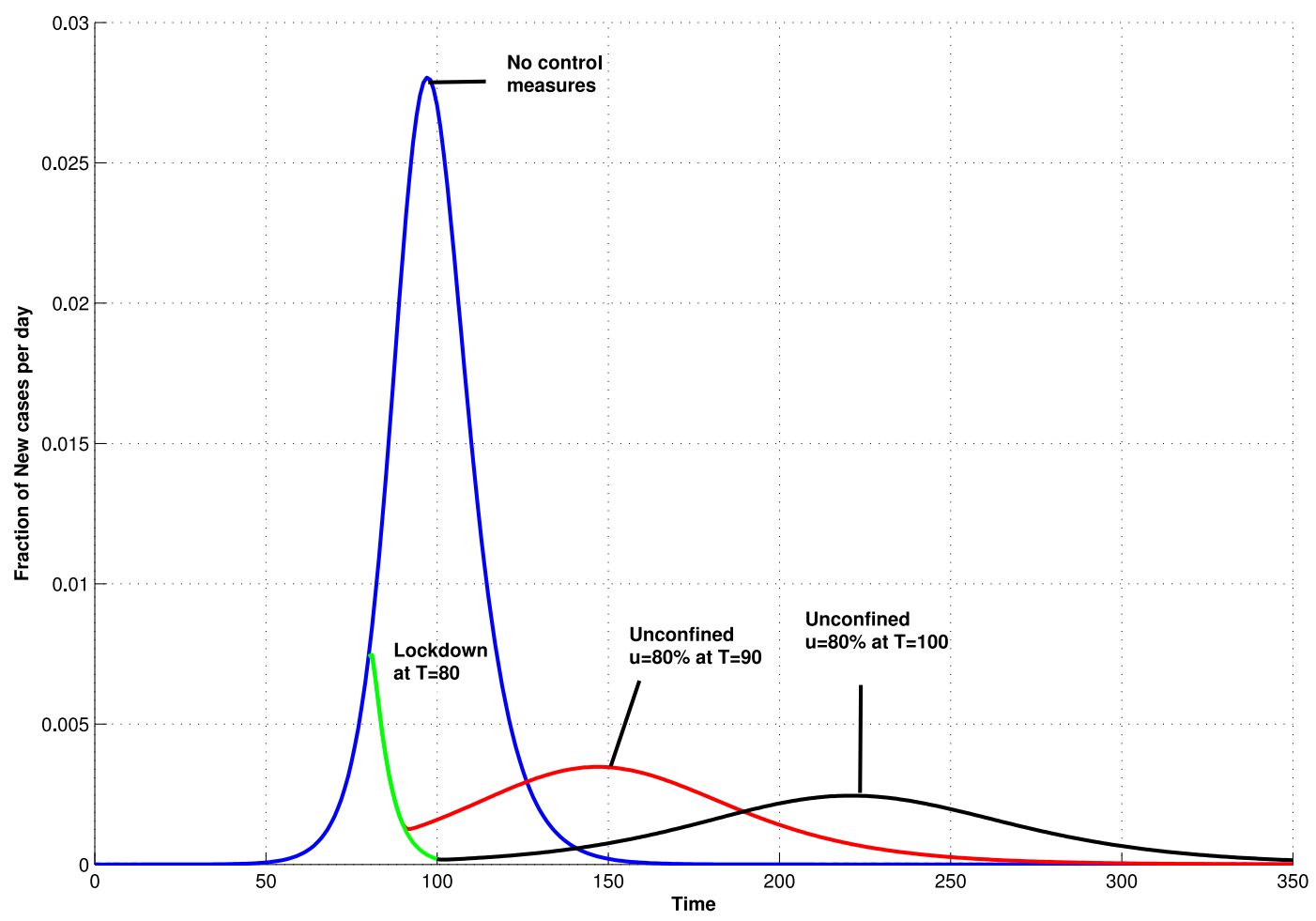

(c)

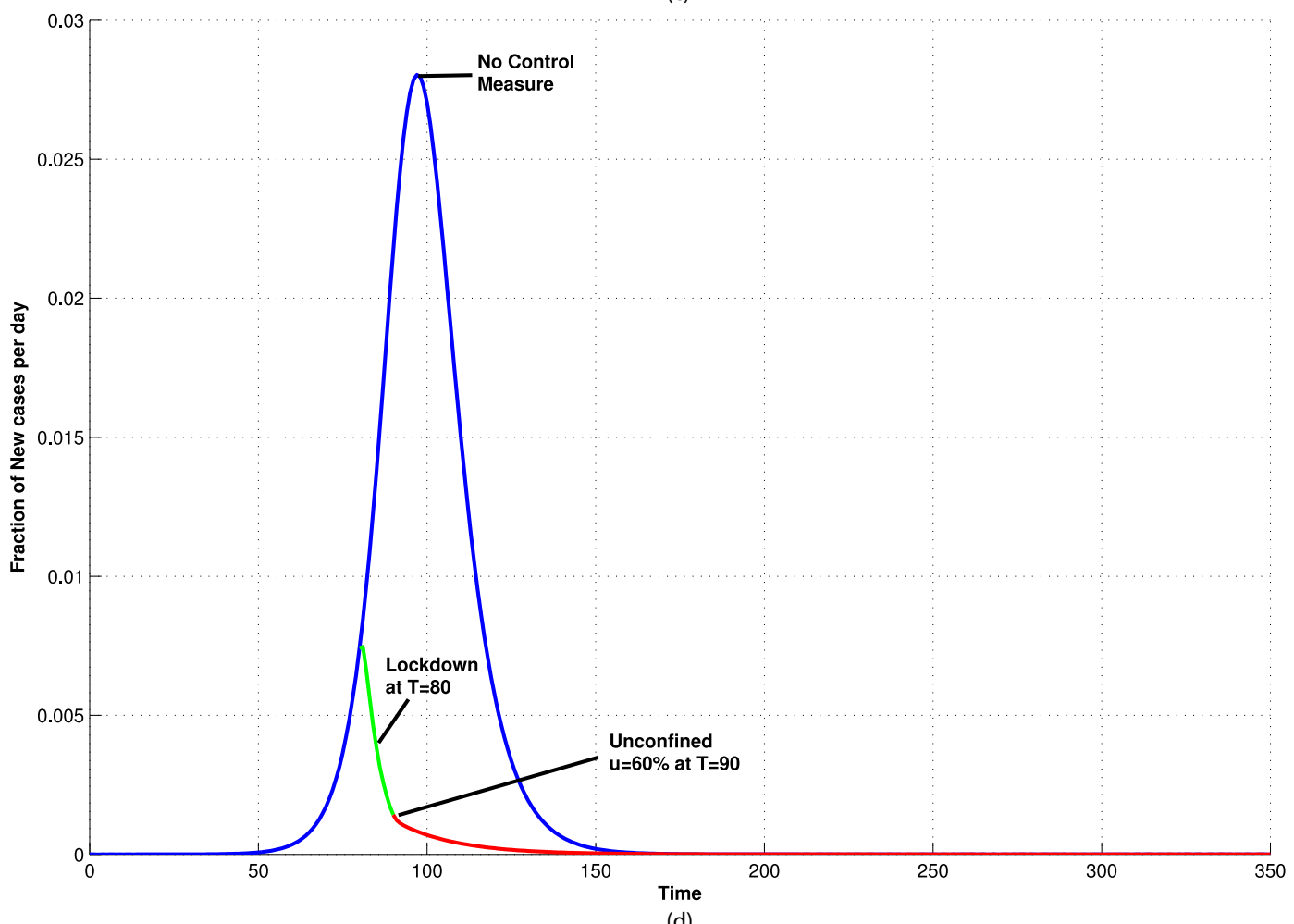

Figure 6. Continued. 
The confinement might also focus on persons who are not very involved in the economic activity (aged, retired, scholars, students, teachers...) and those who can do teleworking. The confinement in each cluster should be done at the maximum level $v=1-u$ that would maintain a good level of the economic activity and allowing to avoid a second peak.

Our model aims to provide information on the dynamics of the epidemic in Algeria. It also makes it possible to study in general the effects of confinement policies and what is very important and current of the measures to be taken for deconfinement. Our model is very simplified compared to reality. It models the set of individual behaviors by that of a super individual type who is believed to be representative of the entire population. We hope, however, that this simplified model provides insights and information for decision-makers.

In perspective, we would like to take into account individual behavior, [13], with individual movements in various places where the risks of contagion are different. For this, it would be necessary to consider several compartments $S_{1}$ instead of just one. Taking into account age classes also seems very important.

Acknowledgements. The authors would like to thank the anonymous reviewers for their valuable comments and suggestions, which have led to a significant improvement of the whole manuscript. This research was supported by the Algerian General Directorate for Scientific Research and Technological Development (DGRSDT).

\section{REFERENCES}

[1] P. Auger, R. Bravo de la Parra, J.C. Poggiale, E. Sanchez and T. Nguyen Huu, Aggregation of variables and applications to population dynamics, in Structured Population Models in Biology and Epidemiology, edited by P. Magal, S. Ruan. Lecture Notes in Mathematics, Mathematical Biosciences Subseries, 1936. Springer, Berlin (2008) 209-263.

[2] P. Auger, R. Bravo de la Parra, J.C. Poggiale, E. Sanchez and L. Sanz, Aggregation methods in dynamical systems variables and applications in population and community dynamics. Phys. Life Rev. 5 (2008) 79-105.

[3] N. Bacaër, Un modèle mathématique des débuts de l'épidémie de coronavirus en France. MMNP 15 (2020) 29.

[4] F. Brauer and C. Castillo-Chavez, Mathematical Models in Population Biology and Epidemiology, 2nd edn. Springer, Berlin (2012).

[5] O. Diekmann, J.A.P. Heesterbeek and J.A.J. Metz, On the definition and the computation of the basic reproduction ratio R0 in models for infectious diseases in heterogeneous populations. J. Math. Biol. 28 (1990) 365-382.

[6] A. Hillion, Les Théories mathématiques des populations. Presses Universitaires de France, Paris (1986).

[7] Wikipédia, Available from: https://fr.wikipedia.org/wiki/Pandémie_de_Covid-19_en_Algérie.

[8] T. Kuniya, Prediction of the Epidemic Peak of Coronavirus Disease in Japan. J. Clin. Med. 9 (2020) 789.

[9] N.M. Linton, T. Kobayashi, Y. Yang, K. Hayashi, A.R. Akhmetzhanov, S. Jung, B. Yuan, B.R. Kinoshita and R.H. Nishiura, Incubation period and other epidemiological characteristics of 2019 novel coronavirus infections with right truncation: A statistical analysis of publicly available case data. J. Clin. Med. 9 (2020) 538.

[10] Z. Liu, P. Magal, O. Seydi and G. Webb, Understanding Unreported Cases in the COVID-19 Epidemic Outbreak in Wuhan, China, and the Importance of Major Public Health Interventions. Biology 9 (2020) 50.

[11] J. Ma, Estimating epidemic exponential growth rate and basic reproduction number. Infect. Dis. Model. 5 (2020) $129-141$.

[12] P. Magal and G. Webb, Predicting the number of reported and unreported cases for the COVID-19 epidemic in South Korea, Italy, France and Germany. Preprint MedXiv DOI: 10.1101/2020.03.21.20040154 (2020).

[13] P. Manfredi and A. d'Onofrio, Modeling the Interplay Between Human Behavior and the Spread of Infectious Diseases. Springer, Berlin (2013).

[14] Ministry of Health, Population and Hospital Reform of Algeria. Available from: http://www.sante.gov.dz/ (2020).

[15] X. Rong, L. Yang, H. Chu and M. Fan, Effect of delay in diagnosis on transmission of COVID-19, Math. Biosci. Eng. 17 (2020) 2725-2740.

[16] H. Sun, Y. Qiu, H. Yan, Y. Huang, Y. Zhu and S.X. Chen. Tracking and predicting COVID-19 epidemic in China mainland. Preprint medRxiv DOI:10.1101/2020.02.17.20024257 (2020).

[17] P. van den Driessche and J. Watmough, Reproduction numbers and sub-threshold endemic equilibria for compartmental models of disease transmission. Math. Biosci. 180 (2002) 29-48.

[18] J.S. Weitz and J. Dushoff, Post-death Transmission of Ebola: Challenges for Inference and Opportunities for Control. Sci. Rep. 5 (2015) 8751. 